\title{
Levantamento da micoflora presente em grãos ardidos e sementes de milho
}

\author{
Adalgisa Thayne Munhoz Ramos ${ }^{1 *}$, Maria Heloisa Duarte de Moraes $^{1}$, Roberto Venceslau De Carvalho ${ }^{2}$, Luis \\ Eduardo Aranha Camargo'.
}

${ }^{1}$ Departamento de Fitopatologia e Nematologia, Escola Superior de Agricultura "Luiz de Queiroz", Universidade de São Paulo, Cx. Postal 9, CEP 13418-900, Piracicaba, SP, fax (0xx19) 3434-4839; ${ }^{2}$ Dow AgroSciences Industrial Ltda. Jardinópolis/ SP.

Autor para correspondência: Adalgisa Thayne Munhoz Ramos (thaynemunhoz@hotmail.com)

Data de chegada: 27/11/2008. Aceito para publicação em: 25/06/2010.

1629

\section{RESUMO}

Ramos, A.T.M; Moraes, M.h.d.; Carvalho, R.V. \& Camargo, L.E.A. Levantamento da micoflora presente em grãos ardidos e sementes de milho. Summa Phytopathologica, v.36, n.3, p.257-259, 2010.

$\mathrm{Na}$ cultura do milho as podridões de espigas causadas por fungos destacam-se como uma das principais responsáveis pelas perdas em produção e qualidade, principalmente devido a formação dos chamados grãos ardidos. O objetivo deste trabalho foi o de identificar a micoflora presente em grãos e sementes produzidos em diferentes regiões e zonas macro-climáticas do Brasil, nos períodos de safra e safrinha. A determinação da incidência dos gêneros fúngicos foi feita pelo método do papel de filtro com congelamento. Foram analisados duzentos grãos/ sementes por amostra em um total de 44 amostras de grãos ardidos e 12 de sementes. As análises foram efetuadas sob microscópio estereoscópico e microscópio óptico. Os principais fungos encontrados neste levantamento, tanto em sementes como em grãos ardidos, foram Penicillum sp., Fusarium spp., Aspergillus spp., Cladosporium sp., Cephalosporium sp. e Stenocarpella spp. Dentre os patógenos principais, Fusarium spp. e Penicillium sp. foram os gêneros encontrados em maior incidência tanto nos grãos como nas sementes nas duas safras. Não houve diferenças significativas entre os diferentes climas e regiões na safra verão para a incidência de Fusarium spp. e Penicillium sp. Porém, durante a safrinha, foi observada uma maior incidência de Fusarium spp. na região CO. Cladosporium sp. se destacou entre os fungos sobretudo durante a safrinha.

Palavras-chave adicionais: milho, grãos ardidos, fungos e zonas climáticas

\section{ABSTRACT}

Ramos, A.T.M; Moraes, M.h.d.; Carvalho, R.V. \& Camargo, L.E.A. Survey of mycoflora in grains and kernels of maize. Summa Phytopathologica, v.36, n.3, p.257-259, 2010.

In maize crop, ear rot caused by fungi is one of the main responsible for quality and production losses, mainly because of the development of the so called discolored grains. The purpose of this study was to identify the mycoflora present in grains and kernels produced in different regions and climatic zones of Brazil during summer and winter harvest and relate the occurrence of fungi with such parameters. The identification of the fungi was done by the filter paper with freezing method. Two hundred grains/kernels for sample totaling 44 samples of discolored grains and 12 of kernels were analyzed. The tests were performed under a stereomicroscope and an optical microscope. The main fungi found in this survey, both in kernel and discolored grains were Penicillium sp., Fusarium spp., Aspergillus spp., Cladosporium sp. Cephalosporium sp. and Stenocarpella spp. Among the major pathogens, Fusarium spp. and Penicillium spp. were found in higher incidence in grains and kernels in both seasons. There were no significant differences between regions and climates in the summer season for Fusarium spp. and Penicillium sp. incidence. However, during the winter season there has been observed a higher incidence of Fusarium spp. in the region CO. Cladosporium sp. stood out among fungi especially during the winter season

Keywords: maize, discolored grain, fungi, climatic zone

Grãos atacados por patógenos e/ou que sofreram algum tipo de injúria que leva a alteração de cor, fermentação em toda área do germe ou em qualquer outra parte do endosperma são considerados "grãos ardidos (2), porém em termos fitopatológicos, apenas os grãos infectados por fungos são considerados "ardidos".

Atualmente, os grãos ardidos constituem um dos principais problemas nas lavouras de milho, devido à queda de produtividade $\mathrm{e}$ qualidade dos grãos, resultando na desvalorização da produção. Os agentes causais dos grãos ardidos mais comumente encontrados em milho são: Fusarium, Aspergillus, Penicillium, Cladosporium, Cephalosporium e Stenocarpella (9).
A incidência desses agentes em grãos e sementes de milho representa ainda um problema adicional devido à produção de micotoxinas. No Brasil, os relatos de fungos toxigênicos na cultura do milho apontam a predominância do gênero Fusarium, seguido por Aspergillus e Penicillium (6).

Apesar da importância da problemática, no Brasil, ainda não existem levantamento da presença desses patógenos em sementes e grãos de milho relacionados a diferentes condições climáticas e regiões de cultivo. Desta forma, o objetivo do trabalho foi realizar um levantamento dos principais fungos presentes em grãos e sementes de milho cultivadas nas safras verão e safrinha em diferentes regiões e 
zonas macro-climáticas do país.

Foi realizado um levantamento da sanidade de 44 amostras, de $1 \mathrm{~kg}$ de grãos ardidos e 12 amostras, de $1 \mathrm{~kg}$ de sementes, nas safra verão (2006) e safrinha (2006/2007). As avaliações foram feitas através de uma análise comparativa da micoflora de grãos ardidos e de sementes, em relação às safras, regiões de cultivo e zonas macro-climáticas. Zonas macro-climáticas correspondem à locais com diferentes altitudes e são utilizadas por empresas produtoras de sementes como, Dow AgroSciences e Embrapa, como parâmetros para avaliar o desempenho dos híbridos e orientar o plantio de seus materiais, nas diferentes regiões produtoras de milho do Brasil. Assim, zona macro-climática subtropical alta (SA) engloba locais a uma altitude de 700 metros; tropical alta (TA), locais acima de $700 \mathrm{~m}$; tropical de transição (TT), locais entre 500 e 700 metros e, tropical baixa (TB), locais abaixo de 500 metros (4).

As amostras de grãos ardidos analisadas foram provenientes das regiões Centro-Oeste (CO) (zonas macro-climáticas TA, TB e TT), Sudeste (SE) (zonas macro climáticas TA e SA) e Sul (zonas macroclimáticas TT e SA). E as amostras de sementes, oriundas da Bahia (região Nordeste - NE e zona macro-climática TA) e de Minas Gerais (região Sudeste - SE e zona macro-climática TB). Essa restrição de locais, para as amostras de sementes, foi devido às características desejadas do ambiente para a produção.

A determinação da incidência de fungos foi realizada pelo método do papel de filtro com congelamento (1). Após o período de incubação, fez-se a análise dos grãos sob microscópio estereoscópico e microscópio ótico.

O delineamento experimental utilizado foi o inteiramente casualizado, com quatro repetições de 50 grãos/sementes de cada amostra. A incidência fúngica foi comparada pelo teste de Tukey a 5\% de significância. Os dados originais foram transformados por "x (10).

A incidência de Fusarium spp. e Penicillium sp. nos grãos e sementes foi elevada independente da safra ou região de cultivo (Tabela 1). Esses resultados corroboram com resultados descritos por alguns autores, como Ono et al. (8), que recuperaram esses dois gêneros fúngicos em todas as amostras de grãos que analisaram. Gonzáles et al., (5), avaliando a micoflora de milho na Argentina, também encontraram os patógenos Fusarium spp. e Penicillium sp. como os mais freqüentes, e apontaram Aspergillus spp., Cladosporium sp. e Cephalosporium sp. em menores incidências.

As maiores incidências de Aspergillus spp. foram em amostras de grãos provenientes da zona macro-climática TT na safra verão e da região CO na safrinha. Contudo, não significa que esse patógeno não deva ser motivo de preocupação em outras zonas macro-climáticas e

Tabela 1. Incidência média de fungos isolados de grãos ardidos e sementes, cultivados sob diferentes zonas macro-climáticas e regiões durante a safra verão de 2006 e safrinha 2006/2007

\begin{tabular}{|c|c|c|c|c|c|c|c|c|c|c|c|c|}
\hline \multicolumn{13}{|c|}{ GRÃOS ARDIDOS } \\
\hline \multicolumn{7}{|c|}{ SAFR A VERÃO } & \multicolumn{6}{|c|}{ SAFRINHA } \\
\hline Clima & FUS & PEN & ASP & CLAD & CEP & STEN & FUS & PEN & ASP & CLAD & CEP & STEN \\
\hline SA & $37,1 \mathrm{a}$ & $89,0 \mathrm{a}$ & $38,1 \mathrm{a}$ & $62,7 \mathrm{~b}$ & $16,3 \mathrm{a}$ & $32,1 \mathrm{~b}$ & - & - & - & - & - & - \\
\hline TA & $58,0 \mathrm{a}$ & $87,8 \mathrm{a}$ & $23,0 \mathrm{a}$ & $24,2 \mathrm{a}$ & $20,5 \mathrm{a}$ & $26,3 \mathrm{~b}$ & - & - & - & - & - & - \\
\hline TB & $48,0 \mathrm{a}$ & $92,0 \mathrm{a}$ & $26,8 \mathrm{a}$ & $36,5 \mathrm{ab}$ & $19,5 \mathrm{a}$ & $23,5 \mathrm{~b}$ & $63,1 \mathrm{a}$ & $74,1 \mathrm{a}$ & $33,6 \mathrm{a}$ & $62,6 \mathrm{a}$ & $31,1 \mathrm{a}$ & $15,4 \mathrm{a}$ \\
\hline TT & $39,6 \mathrm{a}$ & $96,4 \mathrm{a}$ & $74,6 \mathrm{~b}$ & $64,6 \mathrm{~b}$ & $16,2 \mathrm{a}$ & $7,4 a$ & $60,3 a$ & $68,9 \mathrm{a}$ & $49,9 \mathrm{a}$ & $74,7 \mathrm{a}$ & $26,0 \mathrm{a}$ & $13,6 \mathrm{a}$ \\
\hline REGI ÃO & FUS & PEN & ASP & CLAD & CEP & STEN & FUS & PEN & ASP & CLAD & CEP & STEN \\
\hline $\mathrm{CO}$ & $56,4 \mathrm{a}$ & $91,5 \mathrm{a}$ & $35,1 \mathrm{a}$ & $26,7 \mathrm{a}$ & $23,2 \mathrm{a}$ & $26 \mathrm{a}$ & $73,0 \quad \mathrm{c}$ & $63,6 a$ & $63,9 \mathrm{~b}$ & 88,4 b & $33,6 \mathrm{~b}$ & $10,1 \mathrm{ab}$ \\
\hline $\mathrm{SE}$ & 51, a & $87,9 a$ & $21,8 \mathrm{a}$ & $40,4 \mathrm{ab}$ & $17,5 \mathrm{a}$ & $18,3 \mathrm{a}$ & $34,0 \mathrm{a}$ & $85,0 \mathrm{a}$ & $13,0 \mathrm{a}$ & $50,0 \mathrm{ab}$ & $12,5 \mathrm{a}$ & $5,0 \mathrm{a}$ \\
\hline SUL & $46,7 \mathrm{a}$ & $89,4 a$ & $40,3 \mathrm{a}$ & 52,2 b & $18,9 \mathrm{a}$ & $29,0 \mathrm{a}$ & $51,5 \mathrm{~b}$ & $79,5 \mathrm{a}$ & $23,5 \mathrm{a}$ & $41,5 \mathrm{a}$ & $21,0 \mathrm{ab}$ & $26,3 \mathrm{~b}$ \\
\hline \multicolumn{13}{|c|}{ SEMENTES } \\
\hline & \multicolumn{6}{|c|}{ SAFR A VERÃO } & \multicolumn{6}{|c|}{ SAFRINHA } \\
\hline Clima* & FUS & PEN & ASP & CLAD & CEP & STEN & FUS & PEN & ASP & CLAD & CEP & STEN \\
\hline $\mathrm{TB}$ & 87,3 & 86,8 & 6,2 & 24,3 & 47,7 & 0,7 & $60,0 \mathrm{a}$ & $46,3 \mathrm{a}$ & $6,0 \mathrm{a}$ & $78,0 \mathrm{a}$ & $40,0 \mathrm{a}$ & $8,7 \mathrm{a}$ \\
\hline TA & - & - & - & - & - & - & $81,7 \mathrm{~b}$ & $38,0 \mathrm{a}$ & $5,7 \mathrm{a}$ & $77,3 \mathrm{a}$ & $42,3 \mathrm{a}$ & $7,7 \mathrm{a}$ \\
\hline REGIÃO* & FUS & PEN & ASP & CLAD & CEP & STEN & FUS & PEN & ASP & CLAD & CEP & STEN \\
\hline SE & 87,3 & 86,8 & 6,2 & 24,3 & 47,7 & 0,7 & $60,0 \mathrm{a}$ & $46,3 \mathrm{a}$ & $6,0 \mathrm{a}$ & $78,0 \mathrm{a}$ & $40,0 \mathrm{a}$ & $8,7 \mathrm{a}$ \\
\hline NE & - & - & _- & - & - & - & $81,7 \mathrm{~b}$ & $38,0 \mathrm{a}$ & $5,7 \mathrm{a}$ & $77,3 \mathrm{a}$ & $42,3 \mathrm{a}$ & $7,7 \mathrm{a}$ \\
\hline
\end{tabular}

Porcentagens seguidas pela mesma letra na coluna indicam que não houve diferenças significativas entre a incidência fúngica e os climas e entre a incidência e as regiões avaliadas sob a mesma safra, pelo teste de tukey a $5 \%$

FUS: Fusarium spp; PEN: Penicillium sp; ASP: Aspergillus spp; STEN: Stenocarpella spp.; CEP: Cephalosporium sp; CLAD: Cladosporium sp. SA: Subtropical Alta; TA: Tropical Alta; TB: Tropica Baixa; TT: Tropical de Transição. CO: Centro-Oeste; SE: Sudeste; NE: Nordeste (-): Não foram analisadas amostras de grãos provenientes das zonas macro-climáticas SA e TA na safrinha e de sementes provenientes de TA e região NE, na safra verão

*Não foi efetuada a análise estatística das amostras de sementes da safra verão devido as amostras serem oriundas de uma mesma região e clima 
regiões, uma vez que Ono et al. (8) relatou que, esse gênero pode ser detectado em baixa incidência em grãos recém armazenados, atingindo a máxima incidência nos grãos após o $10^{\circ}$ mês de armazenamento.

Cladosporium sp., embora seja de importância secundária, apresentou incidência acima de $60 \%$ em grãos provenientes das zonas macro-climáticas SA e TT, na safra verão e, nas zonas TB e TT na safrinha (Tabela 1). E incidência acima de $80 \%$ em amostras cultivadas na região $\mathrm{CO}$ durante a safrinha.

Cephalosporium sp., de forma geral, ocorreu em baixa incidência e apenas, em amostras oriundas da safrinha, foi observada diferenças significativas $(\mathrm{P}=0,05)$ entre as regiões de cultivo (Tabela 1). A menor incidência de Cephalosporium sp. pode ser atribuída à baixa ocorrência natural do fungo ou a competição com Fusarium spp. (11).

O gênero Stenocarpella ocorreu em incidências acima de $20 \%$ na safra verão, nas zonas macro-climáticas SA, TA e TB. Não houve diferenças significativas entre as regiões na safra verão e, na safrinha, a menor incidência encontrada foi na região SE.

$\mathrm{Na}$ análise das sementes, Aspergillus spp. e Stenocarpella spp. foram observados em baixas incidências tanto da safra verão como na safrinha. Cladosporium sp. ocorreu em maior incidência na safrinha. E, nas duas safras, a incidência de Cephalosporium sp. foi acima de $40 \%$ (Tabela 1)

As incidências fúngicas apresentadas no presente trabalho parecem ser padrão para o milho brasileiro, sendo Fusarium spp. e Penicillium sp. os fungos mais freqüentes, seguidos por Aspergillus spp., Cladosporium sp., Cephalosporium sp. e, Stenocarpella spp., como raramente relatada (7).

Apesar da produção de sementes ser conduzida com o uso de maior tecnologia, em relação, às lavouras comerciais destinadas a produção de grãos, foi observada alta incidência nas sementes dos gêneros Fusarium e Penicillium, principalmente na safra verão e, alta incidência de Cladosporium na safrinha.

Sendo o fator climático, considerado um dos principais fatores determinantes na ocorrência de fungos em sementes e grãos (3), os dados obtidos neste trabalho permitiram comparar as flutuações fúngicas em sementes e grãos, sob diferentes regiões e zonas climáticas. Contudo, ainda é necessário realizar levantamentos em anos subseqüentes a fim de avaliar a flutuação da micoflora em grãos e sementes de ano para ano.

\section{AGRADECIMENTOS}

À empresa Dow AgroSciences pelo fornecimento das amostras e ao Laboratórios de Patologia de Sementes (ESALQ-USP).

\section{REFERÊNCIAS BIBLIOGRÁFICAS}

1. Brasil, Ministério da Agricultura e Reforma Agrária. Regras para Análise de Sementes. Brasília. 1992.

2. Brasil. Portaria n. 11 de 12 de abril de 1996. Estabelece critérios complementares para classificação do milho. Diário oficial da União, Brasília, n. 72, 1996.

3. França Neto, J. B.; Henning, A. A. Diacom: diagnóstico completo da qualidade da semente de soja. Londrina: EMBRAPA-CNPSo, 22 p. (EMBRAPA-CNPSo. Circular Técnica, 10). 1992.

4. Dow Agrosciences Ltda.: Folheto informativo do híbrido $2 \mathrm{~B} 710$. Disponível em:<http://www.dowagro.com/PublishedLiterature/ $\mathrm{dh}_{-} 048 \mathrm{a} / 0901 \mathrm{~b} 8038048 \mathrm{a} 2 \mathrm{c} 9 . \mathrm{pdf}$ ? file pat h=/013 05014.pdf\&fromPage=GetDoc $>$. Acesso em: 10 agosto, 2008 .

5. González, H.H.L., Resnik, S.L., Boca, R.T. \& Marasas, W.F.O. Mycoflora of Argentinian corn harvested in the main production area in 1990. Mycopathologia 130:29-36, 1995.

6. Kawashima, L.m.; Soares, L.M.V. Incidência de fumonisina B , aflatoxinas B , B , G e G, ocratoxina A e zearalenona em produltos de milho. Cîência e ${ }^{2}$ Tecnologia de Alimentos. v.26, n.3. Campinas, 2006.

7. Marasas, W.F.O. Medical relevance of mycotoxins in Southern Africa. Microbiology Aliments Nutrition. v.6. p.1-5. 1988.

8. Ono.E.Y.S.; Sasaki, E.Y.; Hashimoto, E.H.; Hara, L.N.; Corrêa, B.; Itano, E.N.; Sugiura, T.; Ueno, Y.; Hirooka, E.Y. Postharvest storage of corn: effect of beginning moisture content on mycoflora and fumonisina contamination. Food Additives and Contaminants. v. 19, n. 11, p. 1081-1090. 2002.

9. Pinto, N. F. J., Grãos Ardidos em Milho. Circular Técnica. EMBRAPA, Sete Lagoas - MG. Dez. 2005.

10. Pinto, N.F.J.A.; Vergas, E.A.; Preis, R.A. Qualidade sanitária e produção de fumonisina B1 em grãos de milho na fase de précolheita. Summa Phytopathologica, Jaguariúna, v. 33, n. 3, p. 304-306, 2007.

11. Trento, S.M., Irgang, H.H., Reis, E. M., 2002, Efeito da Rotação de Culturas, da Monocultura e da Densidade de Plantas na Incidência de Grãos Ardidos em Milho. Fitopatologia Brasileira, 27 (6), nov - dez 2002. 